\title{
Molecular characterization of signalling pathways in cancer stem cells
}

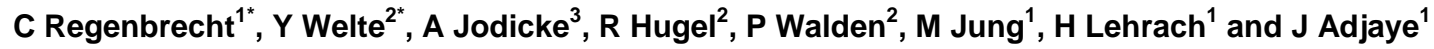 \\ ${ }^{1} \mathrm{MPI}$ for Molecular Genetics, Berlin, Germany \\ ${ }^{2}$ Clinical Research Group tumour Immunology, Department of Dermatology, Charite'-Universitatsmedizin, Berlin, Germany \\ ${ }^{3}$ Clinic for Neurosurgery, Vivantes Klinikum Neukolin, Berlin, Germany
}

*These authors contributed equally to this work

\begin{abstract}
To avoid artefacts introduced by culturing cells for extended periods of time, it is crucial to use low-passage patient-derived tumour cells. The ability to enrich, isolate and assay sub-populations of cells that behave as cancer stem cells (CSCs) from these primary cell lines is essential before performing characterizations such as gene-expression profiling. We have isolated cells from glioblastomas which show characteristics of CSCs. Although glioblastomas contain only a relatively small amount of putative CSCs, these cells express many genes which seem to be worthy targets for future therapies.
\end{abstract}

Published: 02/12/2008

Received: 15/10/2008

ecancer 2008, 2:115 DOI: 10.3332/ecancer.2008.115

Copyright: (c) the authors; licensee ecancermedicalscience. This is an Open Access article distributed under the terms of the Creative Commons Attribution License (http://creativecommons.org/licenses/by/2.0), which permits unrestricted use, distribution, and reproduction in any medium, provided the original work is properly cited.

Competing Interests: The authors have declared that no competing interests exist.

Correspondence to C Regenbrecht. Email: regenbre@molgen.mpg.de 


\section{Introduction}

Al-Hajj et al were the first to identify and prospectively isolate a minority sub-population of cells from human solid tumours that contained all of the in vivo tumour-forming abilities. The tumourigenic cell population was identified based on its cell surface phenotype. This population could initiate tumours in immuno-compromised mice with as few as 200 cells, while as many as 500,000 or more of the remaining cells in the tumour did not initiate new tumours in mice [1].

Fang et al have shown that upon culturing of metastatic melanoma cell suspensions under appropriate conditions, a subset of cells could be propagated as non-adherent spheres, which could then be induced to differentiate in vitro and to generate tumours in vivo [2]. The ability to acutely isolate and assay sub-populations of cells from tumours that behave as cancer stem cells (CSCs) is essential before performing characterizations such as gene-expression profiling, to avoid artefacts introduced by culturing cells for extended periods of time.

\section{Results}

\section{Tissue samples}

Glioblastoma (GBM) tissues were obtained intra-surgically. A trained pathologist evaluated stage and grading of the removed tumour tissue. For our experiments, we only cultivated cells from tissues containing at least $90 \%$ tumour cells as determined by H\&E staining (Figure1A-C). In a more elaborate diagnosis, we determined glial origin (GFAP) (Figure1D), mitotic activity (KI-67) (Figure1E) and neoplastic character (MAP2C) (Figure1F).

\section{In vitro generation of neurospheres}

Tissues that met our criteria were used for low-passage cell culture. We enriched CSCs using embryonic stem (ES) cell medium supplemented with FGF2 [3]. Cells cultured under these conditions began to change their morphology towards a colony-forming phenotype (Figure 2). This phenotype was similar to that of hES cells cultured under the same conditions. Further analysis showed that the phenotype remained stable for at least four weeks and was reversible by FGF withdrawal.
To show the de-differentiation towards a more primitive neural phenotype, we performed immunofluorescence microscopy of cells from the normal medium and ES medium with NESTIN.

\section{Cell sorting}

To investigate if the treated cells were enriched for putative cancer stem cells, we employed magnetic sorting with a beadcoupled anti-CD133 antibody. Enrichment of CSCs by selective cell culture methods resulted in higher yields of CD133+ cells (Figure 4A). This enrichment was time dependent, with its maximum yield after 16 days of stimulation (Figure 4B).

To determine the quality of enrichment, we kept the cells from the positive and negative fractions in culture for four days and stained them with a fluorescence coupled anti-CD133 antibody (Figure 5).

\section{Pathway analysis}

To identify the mechanisms and pathways underlying CSCs, we conducted RNA-expression analysis using the Illumina platform. For expression analysis, we used CD133+, CD133- and bulk cells from the GBM1207 cell line and compared this data to the expression profile of hES lines $\mathrm{H} 1 / \mathrm{H} 9$ (Figure 6). Expression analysis revealed a group of 11 genes that were overexpressed and known to play a role in the progression of various malignancies (Figure 7).

We validated the differential expression of eight selected marker genes from the expression analysis by real-time PCR (Figure 8). All markers were found significantly (>1.5-fold) overexpressed in CD133+ cells compared to the respective negative fraction.

\section{Conclusion}

Still only little is understood about the origin and nature of CSCs [4]. From our findings, we conclude that although GBMs contain only a relatively small amount of putative CSCs, these cells express many genes, which seem to be worthy targets for future therapies. Among these genes, we found important signalling molecules for angiogenesis, apoptosis, multi-drug resistance and metastasis. A large number of markers that have clinical relevance for the evaluation of prognosis in various tumours show increased expression in CD133+ cells compared to their 

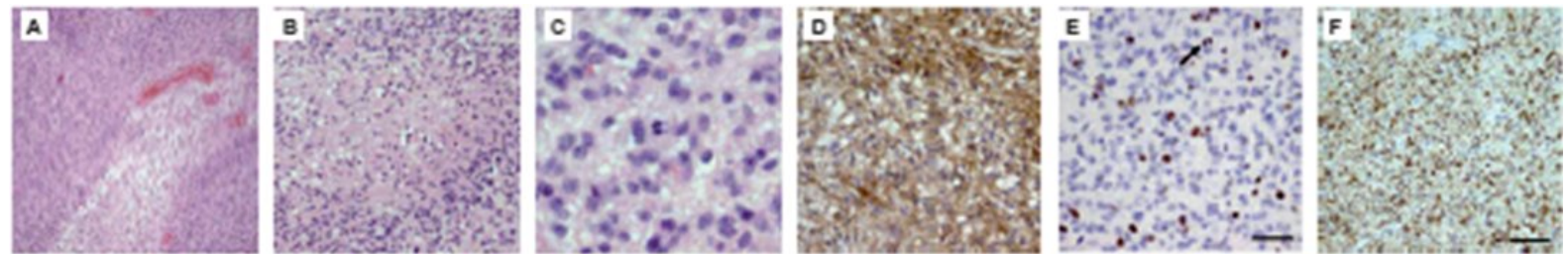

Figure 1: H\&E staining of glioblastoma tissues shows typical histological features of the tumour (A-C). In (A), vessel proliferates are present; (B) shows an area of extensive necrosis, while (C) displays mitotic cells within the tumour. Immunohistochemical characterization of the removed tissue confirms the diagnosis. (D) shows staining against GFAP, a marker for glial origin. (E) KI-67 antibody is used to show the high mitotic activity in the tumour tissue. (F) Proof of neoplastic character of the tissue, using an anti-MAP2C antibody.

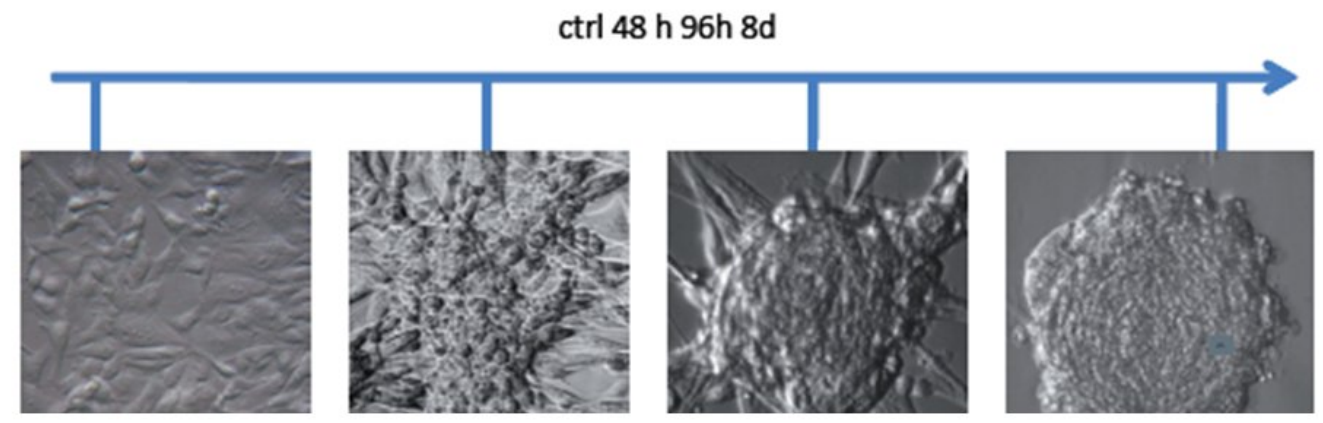

Figure 2: Phase contrast micrographs from human GBM cells treated with ES-medium + FGF2. Untreated cells cultivated in normal medium grew as monolayers. Under ES cell conditions, the cells began to change their morphology and developed a spherical, colony forming phenotype. After eight days of cultivation under these conditions the phenotype remained stable and the spheres began to detach from the substrate.
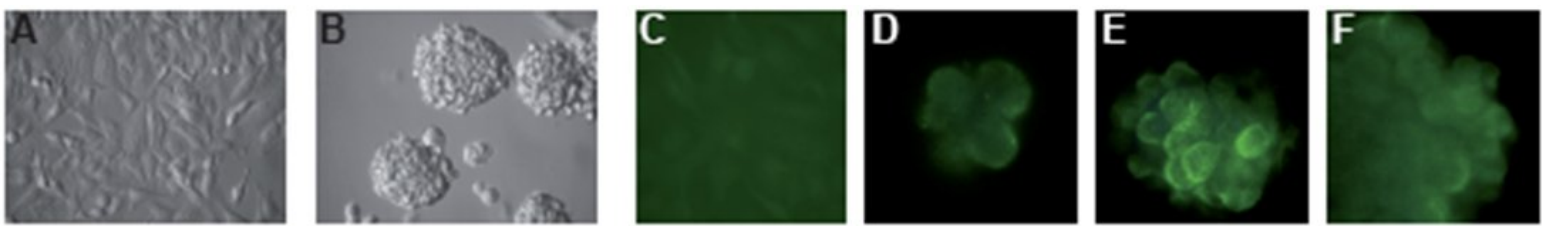

Figure 3: We stained both, adherent monolayer (A) and colony (B) grown cells against Nestin. Nestin is a type VI intermediate filament, which is commonly used as a marker for neuronal precursor cells. Untreated cells do not express Nestin (C), while it was expressed in all spheres (D-F). 

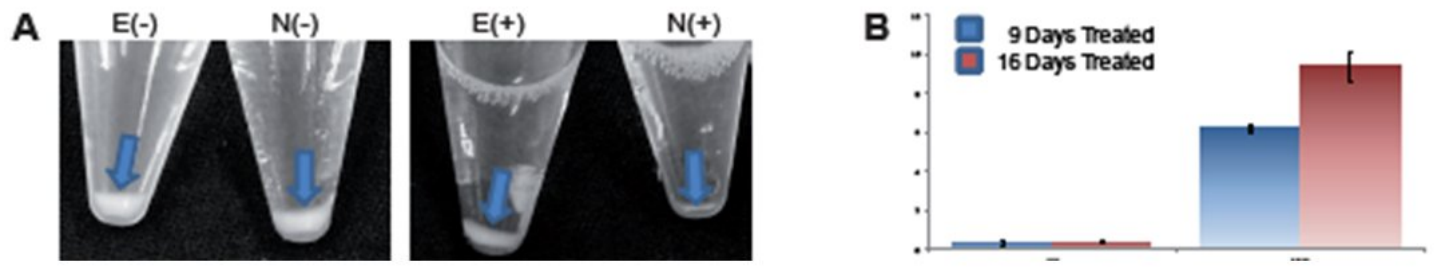

Figure 4: (A) shows the different yields of CD133- and CD133+ cells after MACS sorting. E(-): negative fraction from ES medium; N(-): negative fraction of cells cultured in normal medium; $\mathrm{E}(+)$ : CD133+ fraction of cells cultured in ES medium; $\mathrm{N}(+)$ CD133+ cells isolated from cells grown in tumour medium. The yield of CD133+ cells increases upon duration of stimulation. (B) shows the quantification of CD133+ cells after nine and 16 days of stimulation in ES medium.
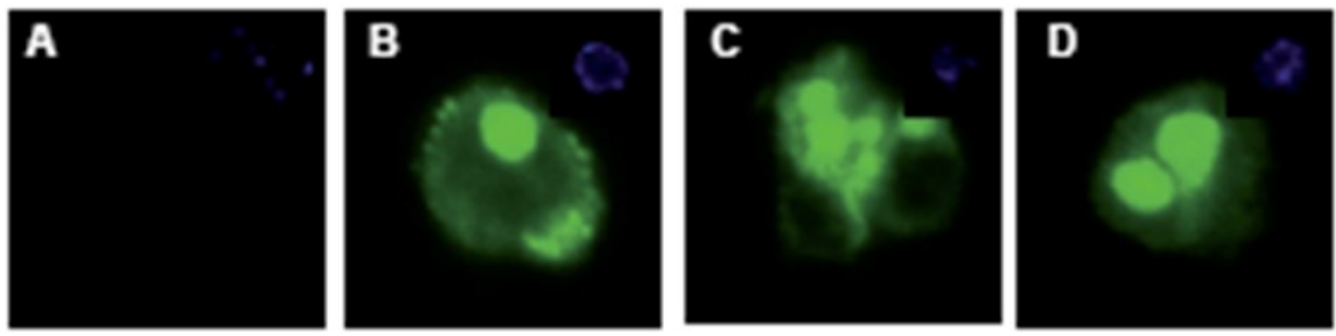

Figure 5: CD133+ and CD133- cells were stained for CD133 expression. The negative fraction (A) showed no CD133 expression, while the majority of cells from the CD133 positive fraction had strong expression of the protein (B-D). DAPI inset.
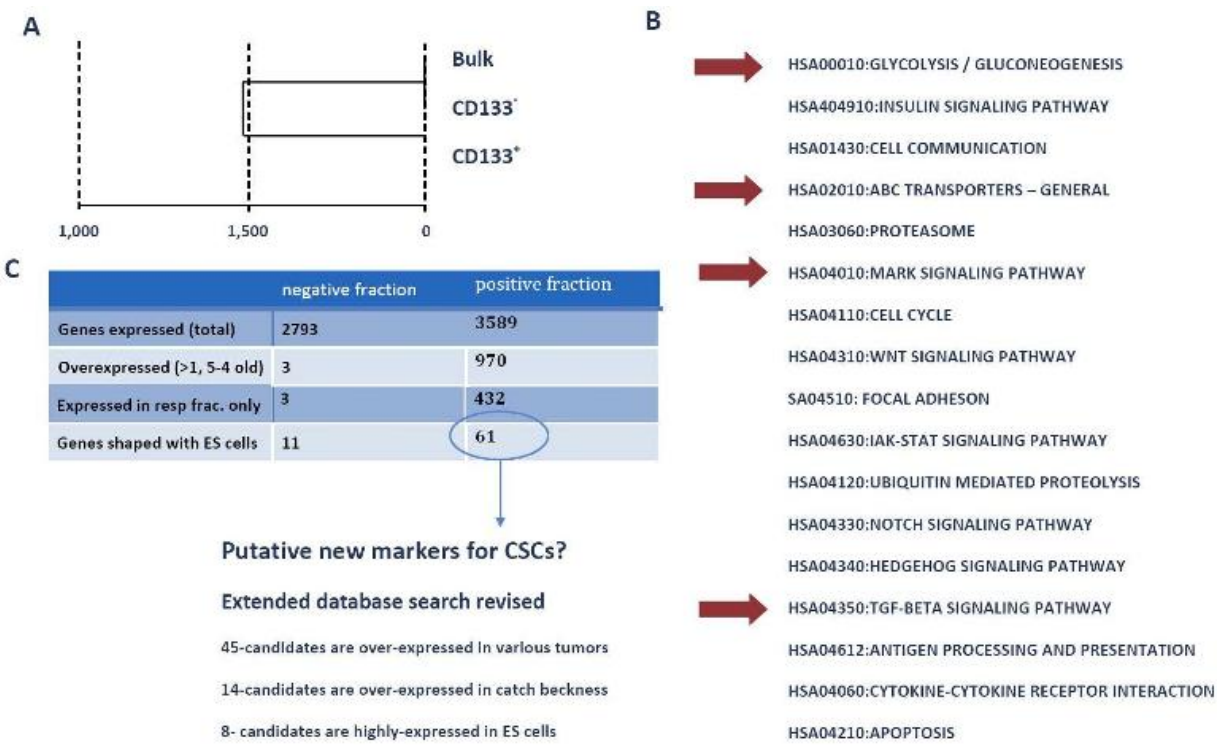

Figure 6: (A) Cluster analysis of expression profiling from GBM1207 bulk, CD133+ and CD133- cells. (B) GO- and KEGG analysis of CD133+ revealed a set of activated pathways, known to be important for oncogenesis. Although most tumours harbour only a small subset of CSCs, these cells might have a high impact on the properties of the tumour. Red arrows mark pathways, which came up in the analysis of CD133+ 
cells and hES cells. (C) Comparison of CD133+l- fraction with human ES cells. The negative fraction shares only 11 genes with hES cells, while 61 genes are expressed exclusively in CD133+ and ES cells. From these 61 candidates, 46 are known to play a role in various cancers and eight targets are most commonly expressed in hES cells. These 61 genes could provide the basis for the identification of new CSC markers and future drugs.

\begin{tabular}{|l|l|}
\hline Gene Symbol & Gene Function \\
\hline PDHA1 & primary link between glycolysis and the tricarboxylic acid (TCA) cycle \\
\hline NCO1 & Neural precursor \\
\hline ABCA1 & Multi-drug resistancy \\
\hline RP130 & Associated with adverse outcome in Modulloblastoma. \\
\hline BMP2 & transforming growth factor-beta (TGFB) superfamily. \\
\hline CK2 & Key mediator for HDAC1 and HDAC2 activation under hypoxia in tumor cells \\
\hline VEGF & Mitogenic, angiogenesis, promoting cell migration, and inhibiting apoptosis. \\
\hline PAI1 & BM-dependent vascularization; BM-indep, tumor growth and angiogenesis. \\
\hline CD99 & Correlates with extent of tumor invasion, mitotic activity, KJ-67 labelling index, \\
\hline CDH1 & Correlation with gastric, breast, colorectal, thyroid and ovarian cancer. \\
\hline IAP & Inhibitor of Apoptosis \\
\hline
\end{tabular}

Figure 7: Among the differentially expressed genes that are shared between CD133+ and hES cells, common regulators of oncogenisis and markers for prognosis were identified. These proteins cover a variety of properties, which include angiogenesis, inhibition of apoptosis, multidrug resistance and cell-cycle control proteins.

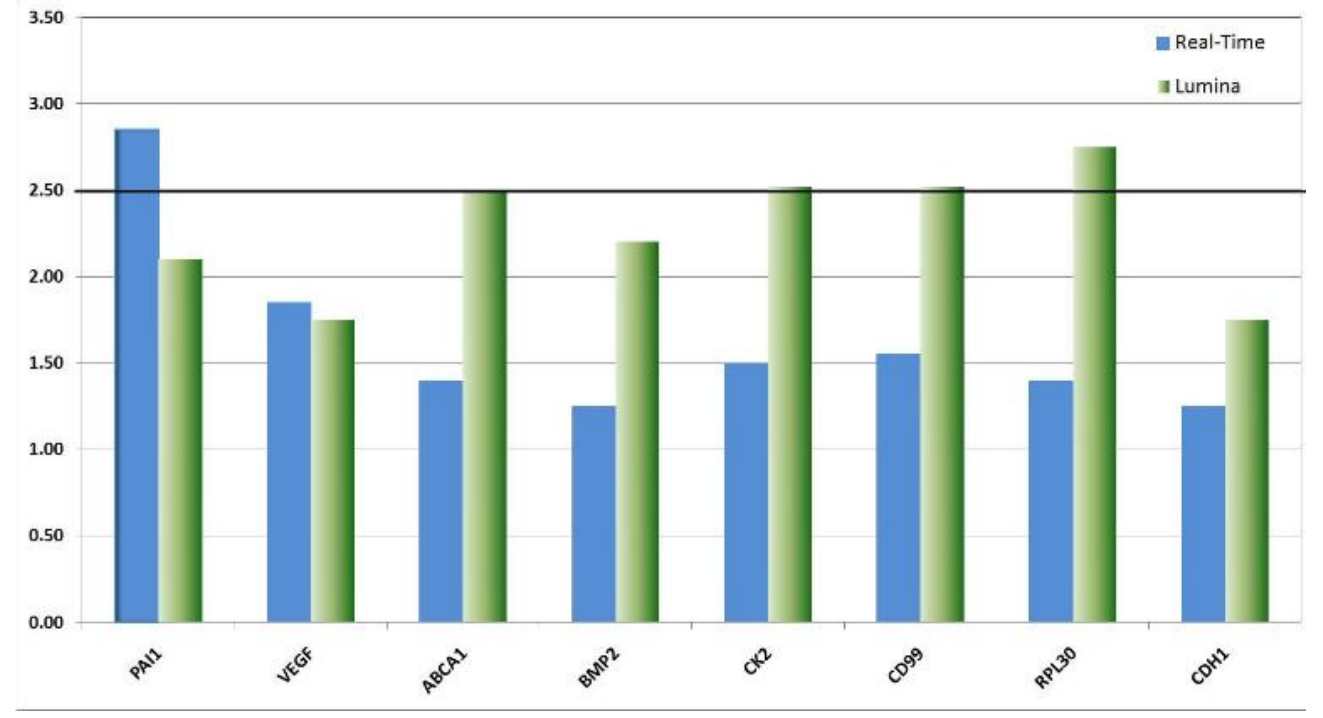

Figure 8: Real-time validation of eight candidate genes identified by genome-wide expression profiling. The relative over-expression of these genes indicates that CSCs despite their rare distribution in the tumour tissue might have a strong influence on the descended cells. The overexpression of genes like VEGF point to the fundamental role CSCs might have on the establishment and maintenance of a tumour promoting micro-environment or niche. Other genes like CK2 function as interfaces in a plethora of cancer relevant pathways such as p53, wnt-signalling and Ç-catenin. 
ecancer 2008, 2:115

CD133- counterparts. Additionally, these CD133+ cells share important pathways with human ES cells, which help explaining self-renewal capacity, drug-resistance and other properties credited to stem cells and cancer.

\section{Acknowledgements}

This work was funded by Gisela-Stadlemann Stiftung, Frankfurt (Main) and Max Planck Society. 


\section{References}

1. Al-Hajj M, Wicha MS, Benito-Hernandez A, Morrison SJ and Clarke MF (2003) Prospective identification of tumourigenic breast cancer cells Proc Natl Acad Sci USA 100 3983-8 PMID: 12629218 doi: 10.1073/pnas. $\underline{0530291100}$

2. Fang D, Nguyen TK, Leishear K, Finko R, Kulp AN, Hotz S, Van Belle PA, Xu X, Elder DE and Herlyn M (2005) A tumourigenic subpopulation with stem cell properties in melanomas Cancer Res 65 9328-37 PMID: 16230395 doi: 10.1158/0008-5472.CAN-05-1343

3. Greber B, Lehrach H and Adjaye J FGF2 Modulates TGF" Signaling in MEF and Human ES cells to support hESC self-renewal Stem Cells 252 455-64 PMID: 17038665 doi: $10.1634 /$ stemcells.2006-0476

4. Regenbrecht $\mathrm{C}$, Lehrach $\mathrm{H}$ and Adjaye J (2008) Stemming Cancer: Functional Genomics of Cancer Stem Cells in Solid tumours Stem Cell Rev 4 319-28 PMID 18561035 